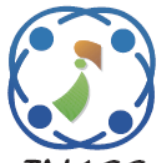

\title{
Coverage Planning for Co-tier Femtocell Networks Using Voronoi Diagram and Gradient-based Optimization Method
}

\author{
Anggun Fitrian Isnawati ${ }^{1} \quad$ Jans Hendry $^{2 *} \quad$ Eko Fajar Cahyadi ${ }^{1,3}$ \\ ${ }^{I}$ Telecommunication Engineering, Institut Teknologi Telkom Purwokerto, Central Java, Indonesia \\ ${ }^{2}$ Departmet of Electrical Engineering and Informatics, Vocational Coll., Universitas Gadjah Mada, Indonesia \\ ${ }^{3}$ Department of Computer Science and Information Engineering, Asia University, Taiwan (R.O.C) \\ * Corresponding author's Email: jans.hendry@ugm.ac.id
}

\begin{abstract}
Effectivity of wireless indoor communication using femtocell network is closely related to the accuracy of the femtocell access point (FAP) placement in each room. Good planning during the FAP deployment process related to the location and the number of FAP needed is the key to creating a more stable indoor communication in accordance with the area, number of users, and distance between FAP and user. Mainly, the methods used herein were Voronoi and gradient-based optimization methods. The aim was to get proportional coverage in term of true coverage, overlaps area, and blank spots area. The simulation was set up with 20-60 FAP units. The result shows that steepest descent and conjugate gradient as complement to proposed methods has successfully outperformed presented existing methods. Their percentage average values of true coverage are $59.3 \%$ and $68.2 \%$, overlaps area are $5.5 \%$ and $10.6 \%$, and blank spots area are $0.0039 \%$ and $0.0007 \%$, respectively.
\end{abstract}

Keywords: Femtocell, Coverage, Optimization, Voronoi, Gradient.

\section{Introduction}

Femtocell deployment can significantly increase the use of radio frequency spectrum. Femtocell can also be easily used by end users based on "plug and play" system [1] and is done by auto configuration [2]. Successful deployment of femtocells requires good planning including efficiently assignment of coverage and interference. The existence of blank spot or overlap can cause problems in user services [3].

Optimization in femtocell deployment including conditions of blank spot, true coverage and overlap areas. The use of some optimization methods to overcome network coverage problems have been carried out by previous researches. Research [4] proposed node coverage optimization method of Ion Motion Optimization (IMO) that compared to Particle Swarm Optimization (PSO), and the result showed that IMO provide the effectively improving convergence speed and coverage rate of nodes. Research [5] compared a proposed coverage optimization method named Directed Randomly Selected Firefly Algorithm (DRSFA) with Firefly Algorithm (FA) and Randomly Selected Firefly Algorithm (RSFA). The results showed that DRSFA can achieve larger network coverage and get better distribution in the shortest time. Other study proposed the Whale Optimization Algorithm (WOA) method that compared to Improved Genetic Algorithm (IGA) and Ant Colony Optimization (ACO) methods. The WOA offered the effectively improving coverage rate of nodes, whole network coverage effect, and leading to prolonging network lifetime [6]. The Improved Grey Wolf Optimizer (IGWO) has also developed to generate wider coverage area compared to original Grey Wolf Optimizer (GWO), PSO, and GA [7]. It was also faster in convergence. Voronoi diagram in some cases of indoor communication planning can also be used as a method of determining the number and location of FAP [8]. Voronoi diagrams are better when used by considering cell areas, and indirectly provide a measure of site density [9]. 
Aforementioned researches solely based their design on overall coverage area i.e. to maximize overall coverage area regardless the presence of overlaps and blank spots. Their aim was to selforganize sensor's location with fixed communication radius. The drawback is that the final solutions were heavily overlapped and led to wider blank spots. This also brought possibility of which some circles had nearly overlapped centre.

This study aims to optimize the femtocell network coverage by taking into account the overlaps and blank spots area. The circles centre was made fixed and distanced by the help of K-Means and Voronoi. Differ to previous researches, it is the circles radius that was optimized to avoid overlaps while providing proportional coverage area. Hence, three metrics were used to measure the quality of proposed scheme were true coverage, overlaps area, and blank spots area.

Organization of this paper is shown as follows. Section 2 explains the co-tier femtocell coverage planning using Voronoi diagram. Research method is described in Section 3. Section 4 shows the results and discussion and then conclusions are given in section 5 .

\section{Co-tier femtocell coverage planning using Voronoi diagram}

\subsection{Indoor communication}

Femtocell increases the quality of coverage to end users of the network indoors. Femtocell is also able to provide more additional services for cellular networks such as multimedia, internet, video calls, voice services, and high-speed data services in a cost effective manner [10]. An example of indoor femtocell communication is shown in Fig.1, of which different Femtocell User Equipment (FUE) can connect to the FAP then use data and voice services.

Cell planning in a building includes planning for coverage according to area, traffic capacity, signal quality, and interference. The most basic thing in indoor communication is the radio system design model and the antenna distribution must be adjusted to the characteristics of the building, that is, the area of the cell coverage is small, the signal is limited to the side of the building, the transmit power used is low, and the size of antenna is small.

\subsection{Co-tier femtocell}

The main challenges in wireless networks come from sources of interference, namely co-tier and cross-tier interference. Co-tier interference refers to

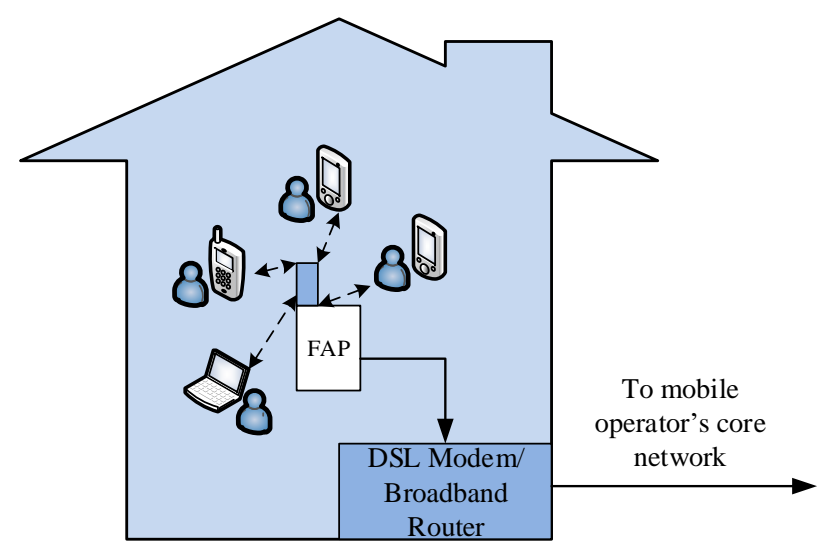

Figure. 1 Indoor femtocell communication [11]

interference among femtocells while cross-tier interference occurs between femtocell and macrocell users. A benefit of femtocells is can be installed by the user, but deployment of femtocells within a macrocell coverage area causes severe co-tier femtocell interferences [12]. Fig. 2 shows unplanned co-tier femtocell configuration as a cause of co-tier interference, while Fig. 3 shows the planned co-tier femtocell configuration with the minimum co-tier interference effect. The two figures show how the severity of the interference increases because of the placement of the adjacent femtocells are too close. The planned femtocell network consists of femtocells whose coverage areas do not overlap with others.

In symmetrical distribution of femtocells, the cotier interference effect becomes smaller due to the natural isolation factor of wall loss. But with the random distribution of femtocells and there may be some extreme scenarios, the impact of co-tier femtocell interference can increase dramatically [13].

\subsection{Voronoi diagram for network planning}

Network coverage map is used to plan the coverage and traffic of communication in the network.

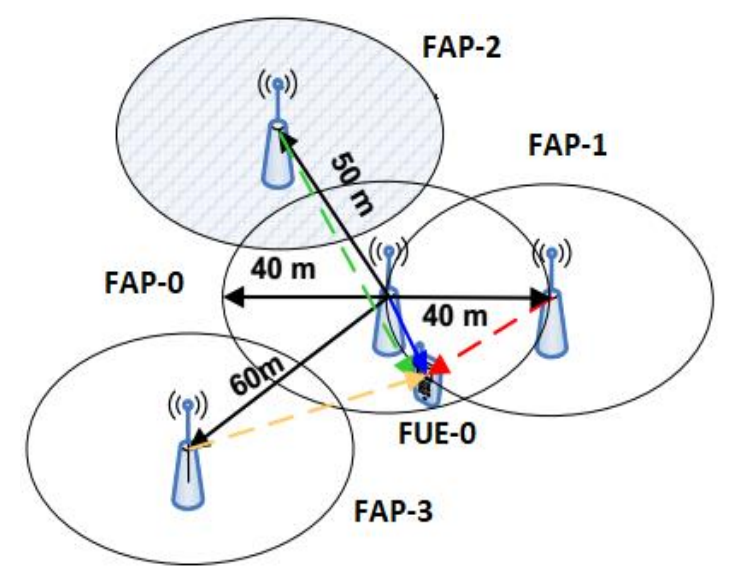

Figure. 2 Unplanned co-tier femtocell configuration [12] 
By superimposing on the city map, geographic data and radio coverage data can be combined, so that some traffic operations such as handoff, registration and outage can be linked to proximity to the location of the base station. The distance between user and base station affects the co-channel interference, frequency reuse, and channel allocation scheme. Spatial information such as terrain, building, and road morphology can be derived from Geographic Information System (GIS), and distance between cells can be derived from the Voronoi diagram. Spatial traffic can be modeled by the proximity relationship between cells. Cell representation using Voronoi diagrams applies to omnidirectional and sectoral cells, as well as hierarchical cell structures [14].

Hexagonal cell size is characterized by site-to-site distance, or Inter-Site Distance (ISD), which is the Euclidean distance between two adjacent sites in perfectly symmetrical structures. Therefore, ISD is a common measure of cellular network density. In practice, however, cellular networks are usually not perfectly hexagonal. Thus, the real network ISD needs to be measured in terms of the actual distance between sites. An alternative characterization of the network density can be extracted from the Voronoi diagram defined by the site positions [9]. From the Voronoi tessellation, it is possible to extract the cellular network area for each site (assuming the signal strength decreases with respect to the distance to the site). This process produces the actual service area size expected from the site. Taking into account the cell area and not just the distance between neighboring sites when characterizing a network, a more complete description can be achieved. This is because the Voronoi diagram can be used for a reasonable estimate of the actual cell size.

\subsection{Diminishing overlaps area}

The FAP coverage area is represented by a circle in $2 \mathrm{D}$ and a sphere in $3 \mathrm{D}$. In order to get wider true coverages, there are two ways that can be adjusted which are optimal locations and radius. Unlike the aforementioned studies, this research proposed a scheme to calculate optimal radius which was initially extracted from Voronoi vertexes and optimized by gradient-based optimization method. The initial radius introduced overlaps area as the circles centre location were made fix and randomly generated using K-Means prior to Voronoi. As the goal to find proportional area amongst true coverage, overlaps, and blank spots then minimizing the overlaps area amongst adjacent circles are done to get

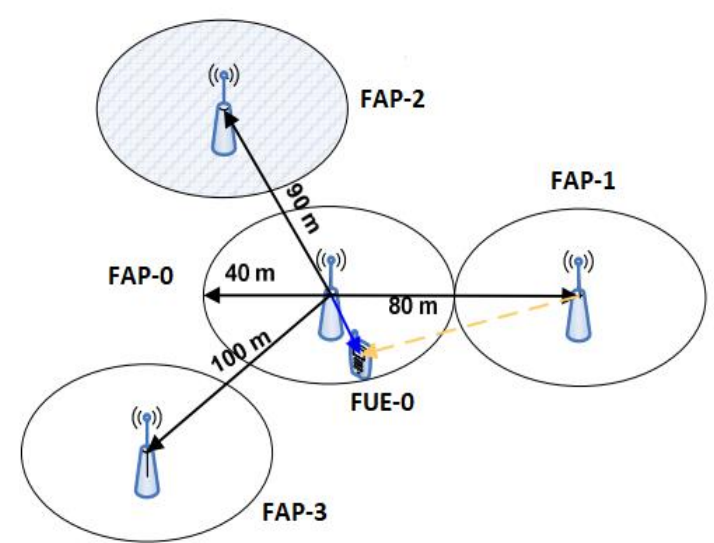

Figure. 3 Planned co-tier femtocell configuration [12]

optimum radius. Added to that, this process becomes a self-organizing.

\section{Research method}

The approach to generate self-organizing femtocell positions by simulating user location is shown in Fig. 4. The K-Means clustering method is conducted prior to Voronoi diagram. It helps to speed up the coverage creation of Voronoi. Herein, four known gradient-based optimization methods were involved and examined to get better description regarding proportional area as previously mentioned. The method that best describes the proportionality is chosen.

\subsection{Users distribution}

A room with size is $\mathrm{M} \times \mathrm{N}$ is considered in this design. A new perspective to this case was being offered of which the room was laid into an image of $\mathrm{M} \times \mathrm{N}$. It means that each pixel can be assumed as users of which real distances among user will be the density of pixels. By doing this, users' positions can be generated randomly based on Poisson distribution [15]. The position set up is done by obeying to Eq. (1).

$$
U_{x y}=\sum_{i=1}^{M^{*} N} \frac{\left(\lambda^{i} e^{\lambda}\right)}{i !}
$$

with $U_{x y}$ is set of generated user positions in 2D space (i.e., based on image pixels), $\lambda$ is mean value, and $i$ is probable occupied location.

\subsection{Initial clustering}

The FAP locations are denoted with $C_{x y}(j)$ which are the centroids calculated by K-Means clustering. Simply, this clustering resembles optimization problem $[16,17]$ as described by Eq. (2). 


\begin{tabular}{|c|c|c|c|c|c|c|}
\hline $\begin{array}{l}\text { Random User } \\
\text { Position } \\
\text { Generation } \\
\text { based on } \\
\text { Poisson } \\
\text { Distribution }\end{array}$ & $\begin{array}{l}\text { Self- } \\
\text { Organizing } \\
\text { Position of } \\
\text { FAP using K- } \\
\text { Means } \\
\text { Clustering }\end{array}$ & $\begin{array}{l}\text { Initialize FAP } \\
\text { Coverage Cell } \\
\text { using Voronoi } \\
\text { Diagram (VD) }\end{array}$ & $\begin{array}{l}\text { Calculate } \\
\text { Circle Radius: } \\
\text { Length of } \\
\text { Farthest and } \\
\text { Nearest Vertex } \\
\text { to Centroid }\end{array}$ & $\begin{array}{l}\text { Use k-NN to } \\
\text { Identify Neighbor } \\
\text { then Calculate } \\
\text { Intersection Area } \\
\text { for Every } \\
\text { Adjacent Circles }\end{array}$ & $\begin{array}{l}\text { Recalculate Each } \\
\text { FAP's Radius By } \\
\text { Minimizing } \\
\text { Largest } \\
\text { Intersection Area } \\
\text { using Gradient } \\
\text { Descent }\end{array}$ & $\begin{array}{l}\text { Use New } \\
\text { Radius Value } \\
\text { to Get } \\
\text { Optimum } \\
\text { Coverage of } \\
\text { Each FAP }\end{array}$ \\
\hline
\end{tabular}

Figure. 4 Proposed approach

$$
C_{x y}(j): \text { minimize } z(W, M)=\sum_{i=1}^{n} \sum_{j=1}^{k} w_{i j} d\left(U_{x y}, \mu_{j}\right)
$$

subject to $\sum_{j=1}^{k} w_{i j}=1$, for $i=1, \ldots, n$

$w_{i j}=0$ or 1 , for $i=1, \ldots, \mathrm{n}$, and $j=1, \ldots, k$. When $w_{i j}$ $=1$ implies object of $U_{x y}$ belongs to $j^{\text {th }}$ cluster and $d\left(U_{x y}, \mu_{j}\right)$ is the distance between $U_{x y}$ and $\mu_{j}$ for $i=1$, $\ldots, n$ and $j=1, \ldots, k$. The distance metric being used herein is cityblock.

\subsection{Initial coverage}

Initially, circle area was calculated based on Voronoi vertexes. The clusters centroid as a result of K-Means clustering is a set of points for Voronoi. Consider an open set $\Omega \subseteq \mathbb{R}$, the set $\left\{V_{i}\right\}_{i=1}^{k}$ is called a tessellation of $\Omega$ if $V_{i} \cap V_{j}=\varnothing$ for $i \neq j$ and $\bigcup_{i=1}^{k} \bar{V}_{i}=$ $\bar{\Omega}$.Given a set of centroids $\left\{C_{x y}(i)\right\}_{i=1}^{k}$ belonging to $\bar{\Omega}$, the Voronoi region $\hat{V}_{i}$ corresponding to the point $C_{x y}(i)$ is defined by Eq. (3) [18].

$$
\hat{V}(i)=\left\{\begin{array}{l}
x \in \Omega|| x-C_{x y}(i)|<| x-C_{x y}(i) \mid \\
\text { for } j=1, \ldots, k, j \neq i
\end{array}\right\}
$$

The set of points $\left\{C_{x y}(i)\right\}_{i=1}^{k}$ is also called as generators. While the set $\left\{\widehat{V}_{i}\right\}_{i=1}^{k}$ is Voronoi tessellation or Voronoi diagram of $\Omega$, and each $\widehat{V}_{i}$ is referred to as the Voronoi region corresponding to $C_{x y}(i)$.

\subsection{Minimum and maximum radius}

The Voronoi diagram yields sets of vertexes that can be used to draw edges surrounding FAP's location. The distances between FAP's locations $\left(C_{x y}\right)$ and their corresponding set of vertexes $(\widehat{V})$ are calculated and stored. Then, minimum and maximum radius of each FAP coverage are calculated by Eq. (4) and Eq. (5) consecutively.

$$
r_{\min }=\underset{C_{x y}(i), \hat{V}(j)}{\operatorname{argmin}}\left(d_{i j}\right)
$$

$$
r_{\max }=\underset{C_{x y}(i), \hat{V}(j)}{\operatorname{argmax}\left(d_{i j}\right)}
$$

with $d_{i j}$ is Euclidean distance between $i$-th centroid and $j$-th vertex.

\subsection{Adjacent search}

Assume that $\Theta_{m}$ denotes a circle represents $m^{\text {th }}$ FAP coverage area and $C_{x y}(m)$ as its centre, then the depleting of overlaps area between adjacent circles require knowledge of neighbour locations. This can be done by using k-NN search. In this research, k-NN employed chebychev as distance metric to get $k$ neighbours around $\Theta_{m}$.

\subsection{Overlaps area calculation}

The overlaps area was diminished by readjusting radius value as such it avoided overlaps. Assume two circles $\Theta_{m}$ and $\Theta_{n}$ has centre $\mathrm{P}$ and $\mathrm{Q}$, respectively. The radius to optimize is $\mathrm{r}_{\mathrm{P}}$ and $\mathrm{r}_{\mathrm{Q}}$ while overlap area is denoted with $L$, over two intersected circles as illustrated by Fig. 5 .

It has been mentioned that the FAP would be placed in a $\mathrm{M} \times \mathrm{N}$ room. This room was laid on an image canvas. As such calculation of coverage area like true coverage, overlaps, and blank spots can be considered in image space. Number of pixels occupies L area represents overlap area. Hence, the metrics calculation can be easily done by creating binary matrix, then filled circles coverage with white pixels and blank spots with black pixels. Then matrix manipulation can be used straightforward.

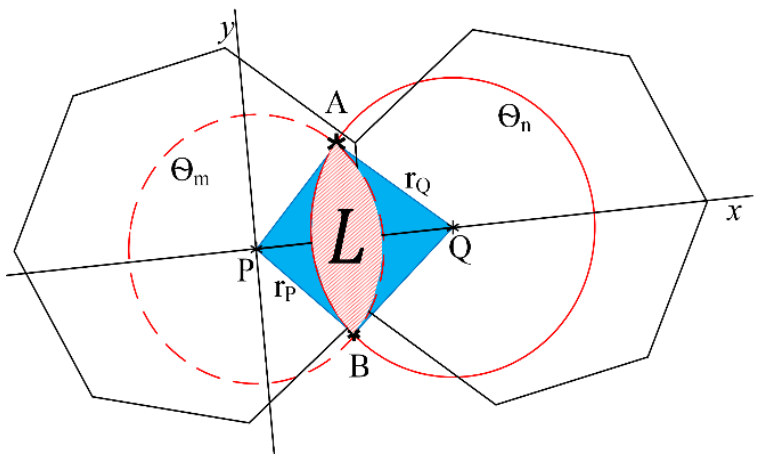

Figure. 5 Illustration of overlap area 


\subsection{Optimal coverage}

Four optimization methods are examined and compared in this simulation. They are Newton, Steepest descent, Conjugate Gradient, and QuasiNewton. In order to update radius value Rosenbrock function was used as expressed in Eq. (6).

$$
f(x)=\sum_{j=1}^{s-1}\left[100\left(x_{j+1}-x_{j}^{2}\right)^{2}+\left(x_{j}-1\right)^{2}\right]
$$

where $s$ is dimension, in this case is 2 , and $x$ is the input. The initial value of $x$ is minimum and maximum radius of each circle. The goal is to estimate optimal radius for all circles. This work employs four methods to approach optimum coverage area as depicted by following mathematic expressions [19]:

1) Newton

$$
x_{i+1}=x_{i}-\mathbf{H}\left(x_{i}\right)^{-1} \boldsymbol{g}_{i}
$$

\section{2) Steepest descent}

$$
x_{i+1}=x_{i}-\alpha \boldsymbol{g}_{i}
$$

3) Conjugate gradient

$x_{i+1}=x_{i}+\alpha_{i} \boldsymbol{\delta}_{i}$

$$
\alpha_{i}=-\frac{\boldsymbol{g}_{i} \boldsymbol{\delta}_{i}}{\boldsymbol{\delta}_{i}^{T} \boldsymbol{Q} \boldsymbol{\delta}_{i}}
$$

4) Quasi-newton

$x_{i+1}=x_{i}+\alpha_{i} \boldsymbol{\delta}_{i}$

$$
\alpha_{i}=\underset{\alpha \geq 0}{\arg \min } f\left(x_{i}+\alpha \boldsymbol{\delta}_{i}\right)
$$

$$
\boldsymbol{\delta}_{i}=-\mathbf{H}_{i} \boldsymbol{g}_{i}
$$

where $\alpha_{i}$ denotes step size, $\mathbf{H}$ is $n \times \mathrm{x} n$ Hessian matrix, $\delta_{i}$ is value that indicates directions, $\boldsymbol{Q}$ is a real symmetric positive definite $n \times n$ matrix, and $\mathrm{x}_{\mathrm{i}}$ is radius. Whereas the gradient of objective function which is denoted with $\boldsymbol{g}_{i}$ is expressed by Eq. (14).

$$
\boldsymbol{g}_{i}=\nabla f\left(x_{i}\right)
$$

Optimum radius is a vector in form of $2 \times 1$. In order to get value in single form which is denoted with $r_{\text {opt }}$, its sample mean is calculated according to Eq. (15).

$$
r_{\text {opt }}=\bar{x}_{i+1}=\frac{1}{s} \sum_{j=1}^{s} x_{i+1}(j)
$$

The optimization method that can yield proportional area amongst three aforementioned criterions will be chosen and compared with other existing methods.

\subsection{Performance evaluation}

In this research, quality performance is measured through following metrics:

1) Overlaps area

$$
L_{i}=\frac{\sum_{j}\left(\Theta_{i} \cap \Theta_{j}\right)}{M x N}
$$

where $L$ denotes overlaps area, $\Theta_{i}$ denotes pixels of circle of which its radius will be calculated and $\Theta_{j}$ denotes pixels of neighbour circles that previously searched by k-NN. All pixels present on the intersections are considered as overlaps pixels and summed then normalized by room size.

2) True coverage area

It is a term that just introduced in this study to measure non-overlap part of circle area. While coverage of a circle that includes overlap is called coverage area which was not a concern in this study. True coverage area is calculated according to Eq. (17).

$$
\mathrm{A}=-L_{i}+\frac{\sum \Theta_{i}}{M x N}
$$

where A denotes true coverage area.

3) Blank spots area

$$
\mathrm{O}=\Psi-\mathrm{A}
$$

where $\Psi$ is room area and $O$ is blank spots area.

All metrics are in normalized unit to make it flexible when used in different room size. The performance of 4 optimisation to strengthen coverage decision of Voronoi in term of optimum radius is compared. Then the proposed scheme will be based on best optimization method. The proposed scheme will be compared with other developed methods based on these metrics. The results are expressed thoroughly hereinafter. The notation list is provided in Table 2 in Appendix A at the end of this paper. 


\section{Result and discussion}

User distribution on 2D space was generated randomly obeying Poisson distribution. Illustration of this is shown in Fig. 6 with 9 clusters. The clusters boundaries were generated using Voronoi diagram theorem. Edges were created from vertexes of Voronoi. Voronoi was fed with clusters centroid and formed varying shape depending on number of clusters. This result is illustrated in Fig. 7.

To be noted that unit of all calculation is in normalized unit. Obviously, clusters area has various shape as Voronoi insisted to provide vertexes that created edges occupying whole area space. Then, minimum and maximum radius was calculated based on the Euclidean distance between closest and farthest vertex from centroid. This gives circles circling each centroid with different area as shown by Fig. 8. It also describes how minimum and maximum radius causes circles intersected.

The result of this process is shown in Fig. 9 (a) (d). Room size used herein is $512 \mathrm{~m} \mathrm{x} 512 \mathrm{~m}$ with rate equals to 0.001 . Newton optimization method highly depends on gradient and Hessian Matrix. In its first iteration, it converges to a radius that disassociates all circles because of their small radius. The consequence is blank spots becomes wider than expected and put proportional of three metrics failed. The steepest decent and conjugate gradient have better proportional result. It is obvious that they are able to manage the circles side by side even though conjugate gradient leaves some circles intersected. But overall, their blank spots area is smaller than Newton method as desired.

Quasi-Newton method has successfully depleted the blank spots significantly. On the contrary, overlaps area are wider than all former method which did not describe proportionality as expected. In the absence of overlapping perspective, this method can be the best choice but would end up on inefficient power used.

\subsection{Finding proper gradient-best method}

It has been said previously that the goal of this research was to find optimum radius that could have proportional area between blank spots and overlaps. It means that overlaps and blank spots area should be minimized. Quasi - Newton method is formidable at providing FAP specification that can have narrowest blank spots area. As can be seen in Fig. 10, its true coverage value tends to decreasing when number of FAP gradually increasing as it also increases overlaps area. On the contrary, Newton method has the narrowest true coverage area compare to the others

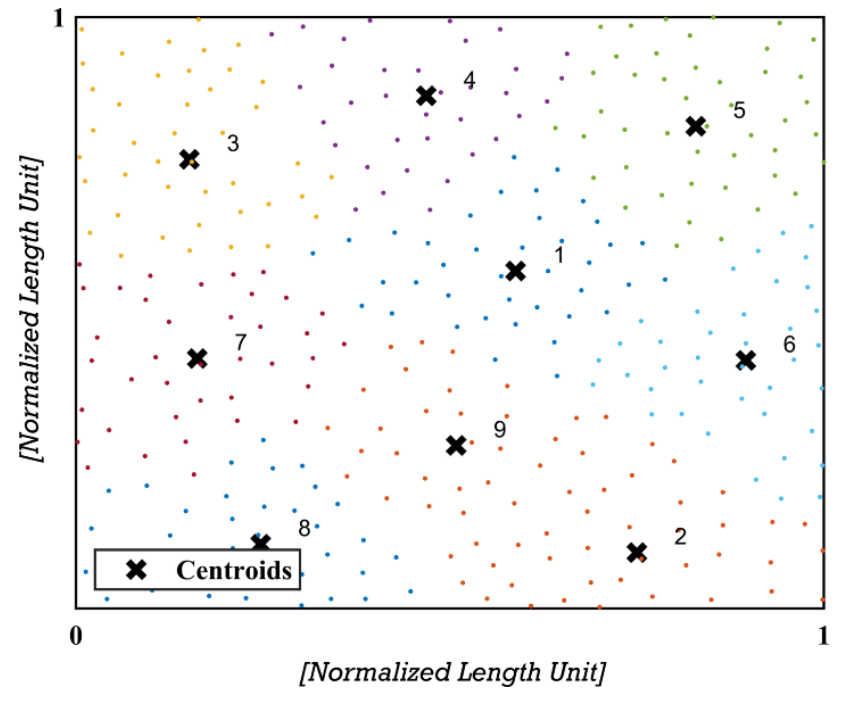

Figure. 6 User distribution illustration with 9 FAPs

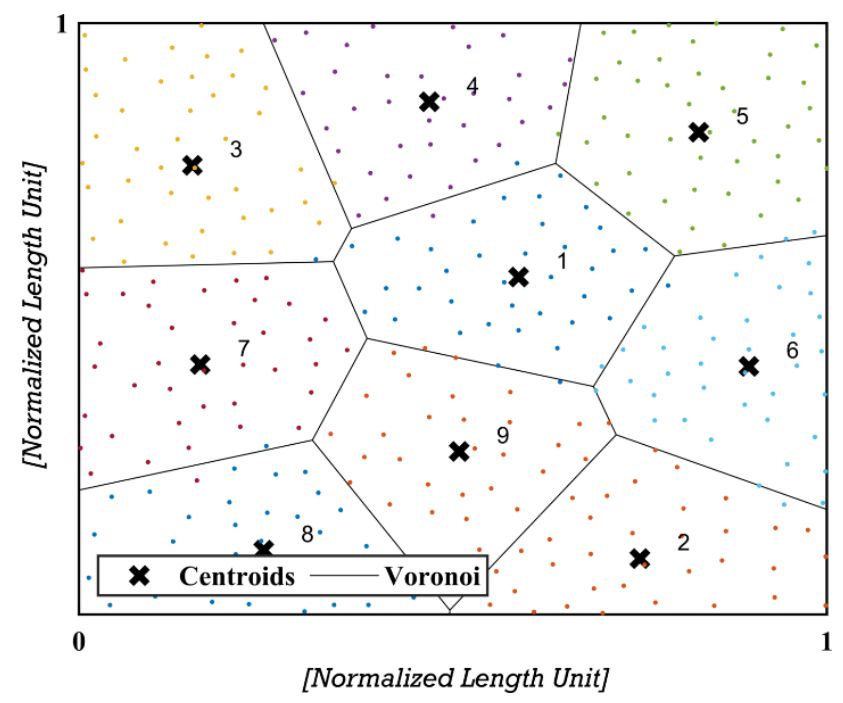

Figure. 7 Voronoi area illustration with 9 FAPs

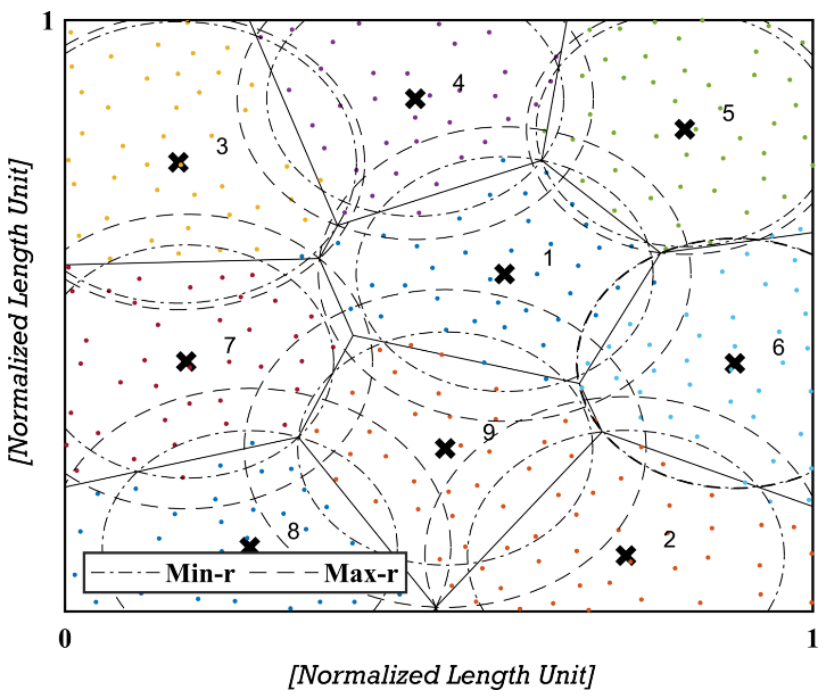

Figure. 8 Illustration of created circles with 9 FAPs 


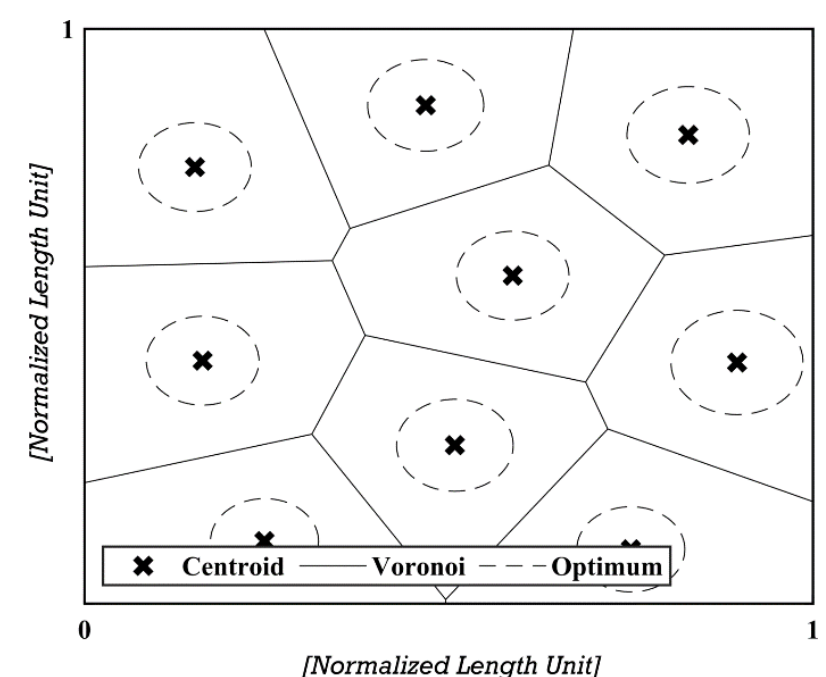

(a)

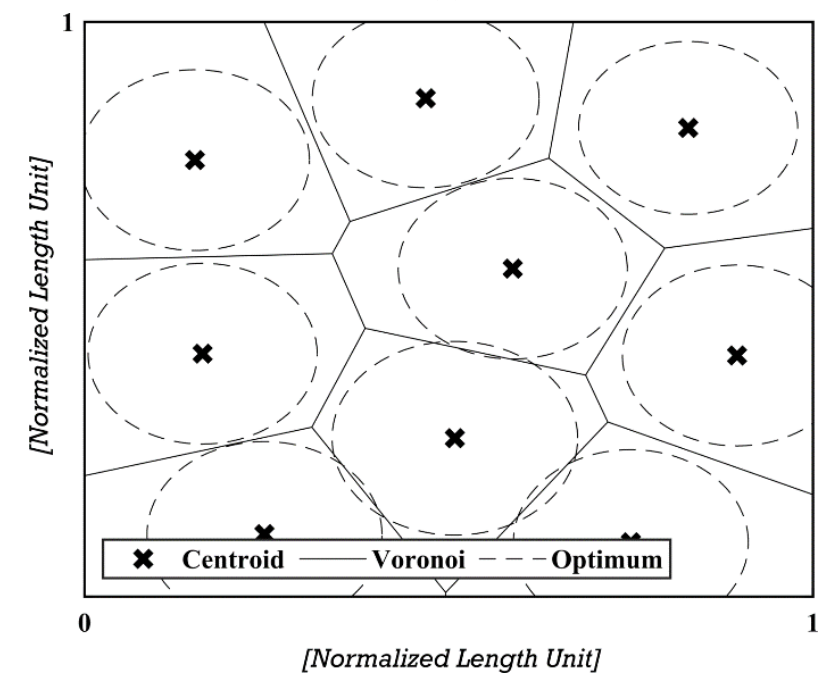

(c)

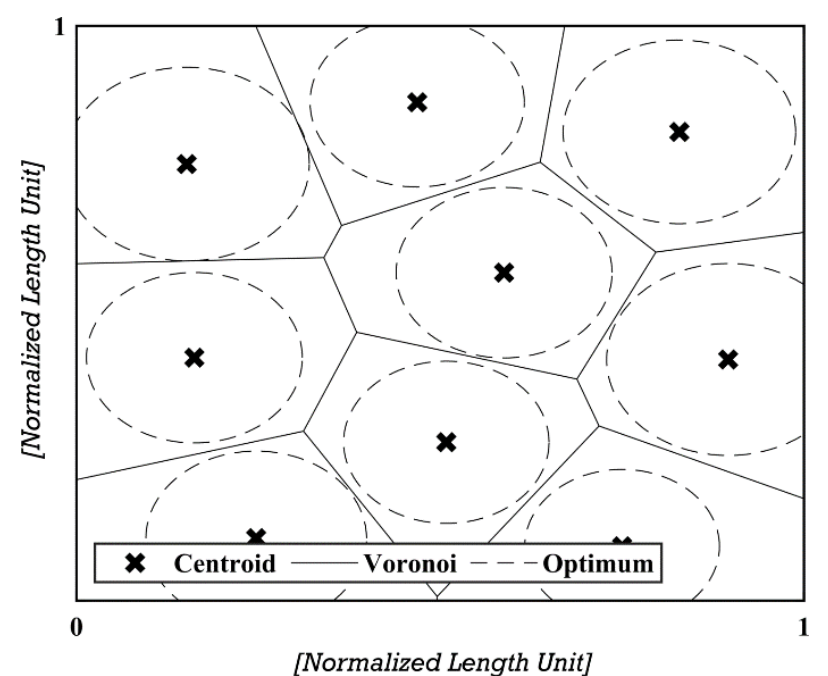

(b)

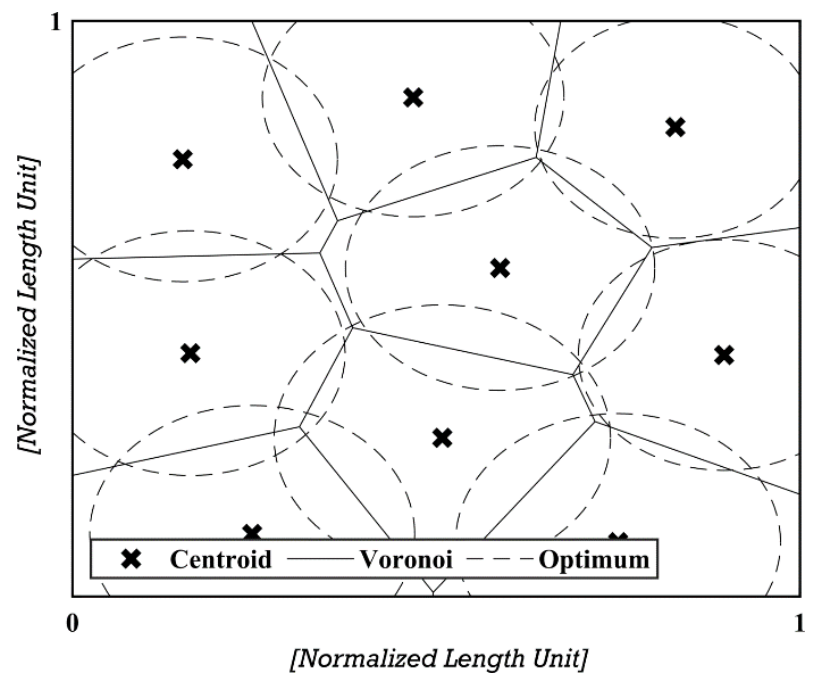

(d)

Figure. 9 Optimization result with 9 FAPs: (a) Newton, (b) Steepest Decent, (c) Conjugate Gradient, and (d) Quasi Newton

and has the widest blank spots area. It is obvious that it does not satisfy the proportionality requirement as well.

Steepest decent and conjugate gradient method provide proportional trade off. Both of them provide pretty wide true coverage area in the range of $0.5-$ 0.8 in normalized unit as depicted in Fig. 10. Moreover, addition of number of FAP will solely swing the curve inside the range. It means that both methods are nearly steady. However, conjugate gradient method is always over the steepest decent but introduces slightly bigger overlaps area as depicted in Fig. 11. Added to that, their blank spots area has shown tendency to slowly decreasing in the range of $0.2-0.5$ in normalized unit when number of FAP increasing as illustrated by Fig. 12. Based on these results, it can be concluded that steepest descent and conjugate gradient will be the proper methods to complete proposed scheme. Further, the scheme that

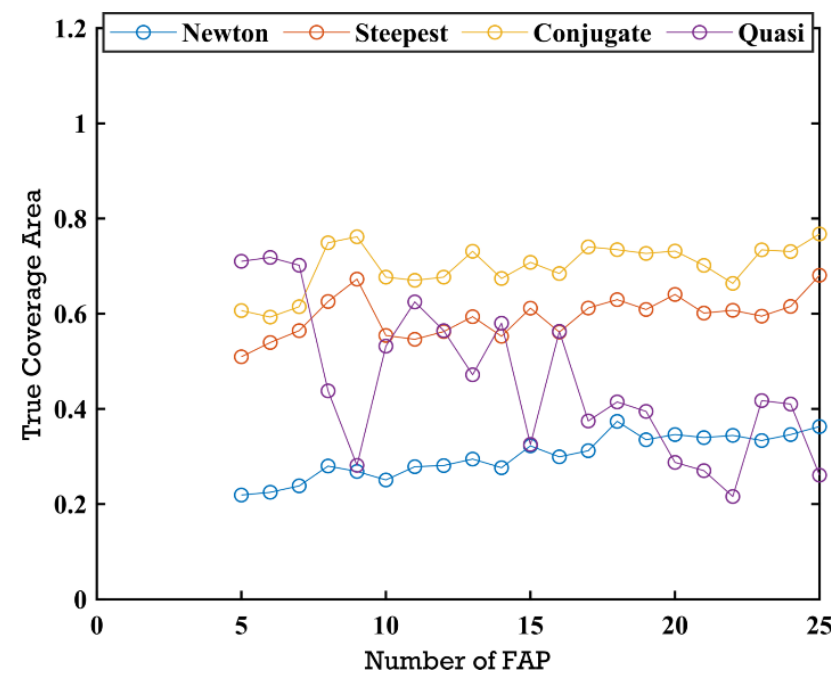

Figure. 10 True coverage area in normalized unit 
uses steepest decent is called proposed-1 while proposed-2 explains scheme that uses conjugate gradient method.

\subsection{Comparison with existing methods}

The proposed methods have been compared with 6 methods which were IMO [4], PSO [4], RSFA [5], DRSFA [5], WOA [6], and IGWO [7]. The circle radius for all methods was set to 0.1 in normalized unit. Room size was set to 512 × 512 similar to proposed methods. Initial solution which is set of circles centre was generated randomly following uniform distribution. Number of FAP used in this comparison is ranging from $20-60$ units.

The comparison results can be seen in Fig. 13, Fig. 14, and Fig. 15. These curves are smoothed versions of real values. They were fitted to second order polynomial because original curves were bumpy. Hence, one can observe tendency of the comparison results. According to Fig. 13, only PSO that outperforms proposed methods but only when number of FAP between $20-45$ units. The true coverage area decreases drastically afterwards. While proposed methods constantly provide steady true coverage area for whole FAP units in the range of 0.6 -0.7 in normalized unit. It implies that there are many overlaps area produced by the existing methods as shown in Fig. 14, the overlaps area of existing methods linearly increases after 25 FAP units and unstoppable. While proposed methods maintain their positions around $0-0.15$ in normalized unit over all FAP units.

Proposed methods outperform existing methods in two metrics. They are not fully better in term of blank spots area. They work best when FAP units less than 40 and tend to stay constant afterwards, while existing methods goes lower when FAP units added more and more as seen in Fig. 15. Optimization methods find optimal radius that can fit in all circles side by side with minimum overlaps area while preserving centre locations. Overall coverage would be ideal if blank spots area and overlaps area nearly zero, simultaneously. Even if this is infeasible but existing methods are better in case installation needs more than 40 FAP units.

Percentage of average value and standard deviation of proposed methods are presented in Table 1. Overall percentage of true coverage is about $59.3 \%$ for steepest decent-based and $68.2 \%$ for conjugate gradient-based method. Even if IGWO is slightly better than the former in this case, but proposed methods expose overlaps area smaller amongst all which are $5.5 \%$ and $10.6 \%$. Their blank spots area is also smaller than other methods which are $46.1 \%$ and

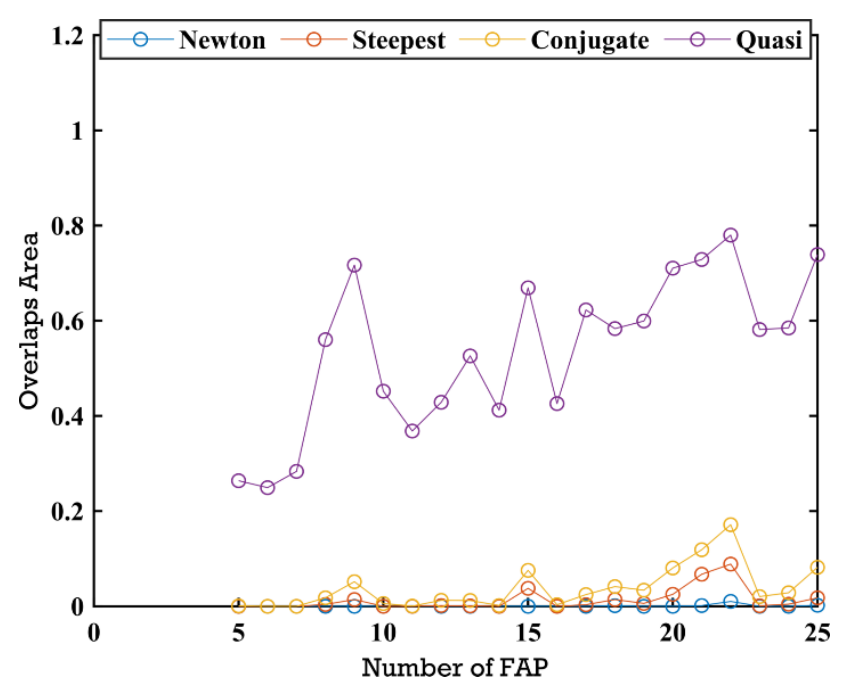

Figure. 11 Overlaps area in normalized unit

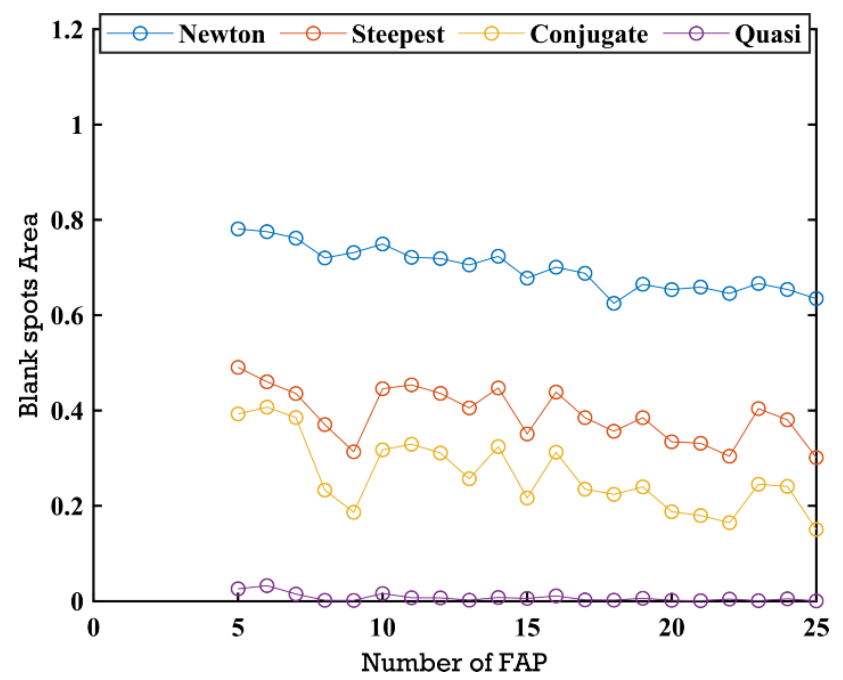

Figure. 12 Blank spots area in normalized unit

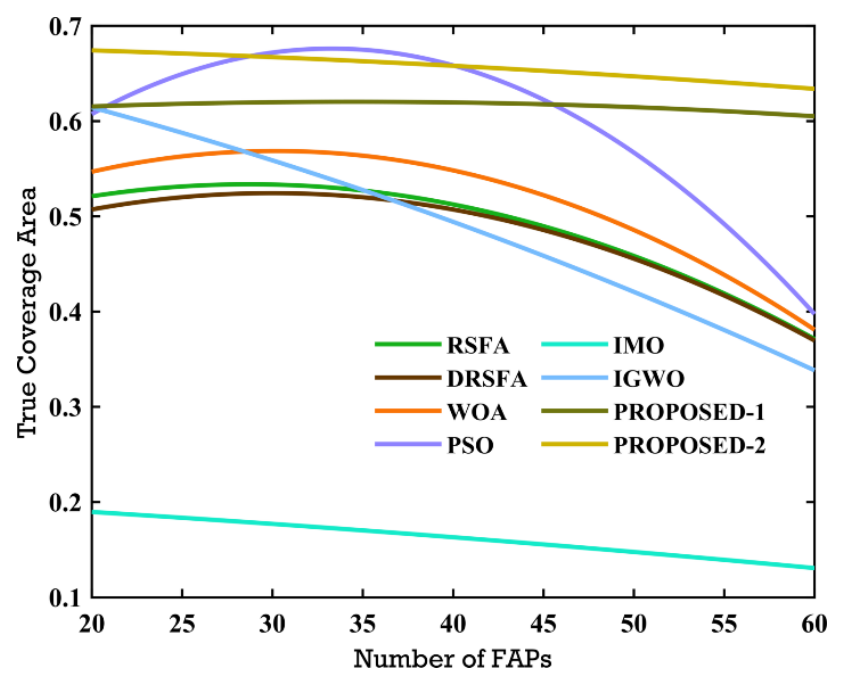

Figure. 13 Comparison of true coverage area

$21.2 \%$. While their true coverage dispersions in term of standard deviation are around $0.0039 \%$ and 


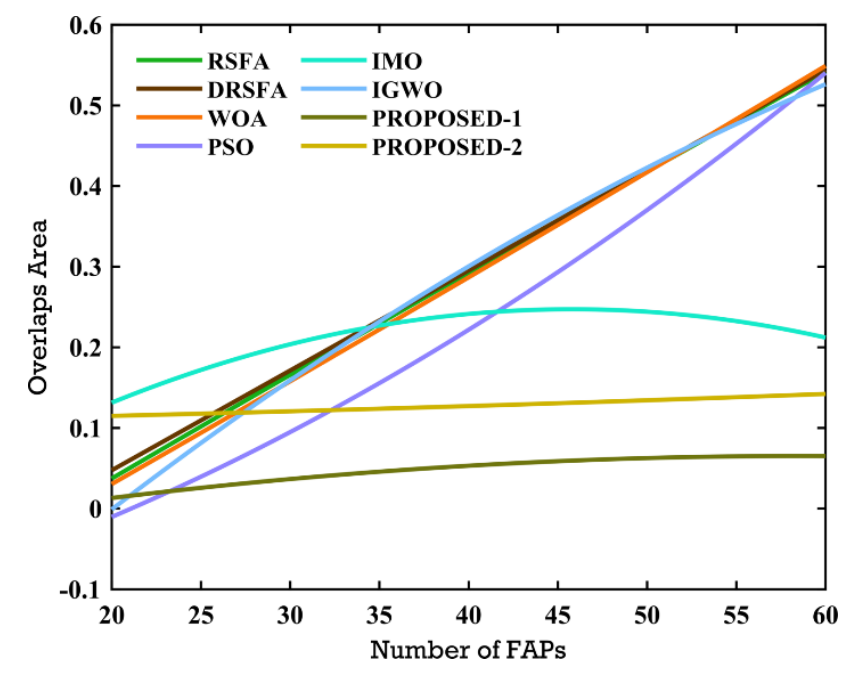

Figure. 14 Comparison of overlaps area

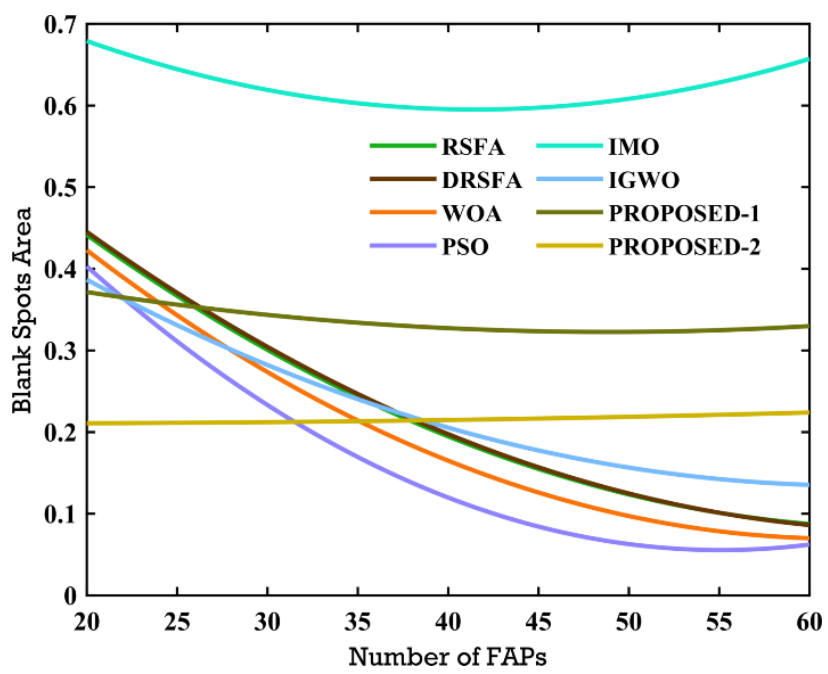

Figure. 15 Comparison of blank spots area

$0.0007 \%$, respectively. It means that no matter how many the FAP number is, both proposed methods can provide better and steady coverage network according to true coverage, overlaps area, and blank spots area metrics. Furthermore, the proposed method with conjugate gradient has the competition over steepest descent in two metrics which are true coverage and blank spots. This method can be said the most match proposed method to proportionality criterion. However, both methods are proposed and left for network planner to choose according to their necessities. The numerical approach has been proposed to bring evidences about their performances and comparisons.

\section{Conclusion}

The simulation has shown that proposed methods that utilize optimization method i.e., steepest descent and conjugate gradient, yielded better coverage area
Table 1. Comparison of average values (\%)

\begin{tabular}{|c|l|r|r|r|}
\hline No & Method & \multicolumn{1}{c|}{ True } & Overlap & \multicolumn{1}{c|}{$\begin{array}{c}\text { Blank } \\
\text { Spot }\end{array}$} \\
\hline 1 & RSFA & 40.2 & 22.1 & 82.3 \\
\hline 2 & DRSFA & 37.5 & 19.6 & 82.4 \\
\hline 3 & WOA & 38.3 & 21.6 & 83.9 \\
\hline 4 & PSO & 26 & 15.5 & 90.7 \\
\hline 5 & IMO & 21 & 11.5 & 89.8 \\
\hline 6 & IGWO & 69.7 & 37.2 & 67.5 \\
\hline 7 & P1 & 59.3 & 5.50 & 46.1 \\
\hline 8 & P2* & 68.2 & 10.6 & 21.2 \\
\hline P1 $=$ Proposed-1 \\
P2 = Proposed- 2
\end{tabular}

network in term of true coverage, overlaps area, and blank spots area compared with presented existing methods. Both methods are better according to 3 metrics, but conjugate gradient wins highest points. Both methods hold constant and steady network area even if number of FAP units added more and more. This is because the radiuses have been optimized to provide proportional occupancy amongst the circles. The overall average values of true coverage of both methods are $59.3 \%$ and $68.2 \%$, overlaps area are $5.5 \%$ and $10.6 \%$, and blank spots area are $0.0039 \%$ and $0.0007 \%$. In the future, self-organizing femtocell placement will be developed where radius and circles centre adaptively changing to get highly proportional network coverage.

\section{Conflicts of Interest}

The authors declare no conflict of interest.

\section{Author Contributions}

Contributions of author are elaborated as follows. Anggun Fitrian Isnawati: conceptualization, resources, writing-original draft preparation, validation and supervision; Jans Hendry: methodology, software, investigation, formal analysis, and visualization; Eko Fajar Cahyadi: administration, writing-review and editing.

\section{Acknowledgments}

This research is fully supported by Lembaga Penelitian dan Pengabdian Masyarakat, Institut Teknologi Telkom Purwokerto (ITTP), Indonesia.

\section{References}

[1] E. Hossain, D. Niyato, and L. B. Le, Radio Resource Management in Multi-Tier Cellular Wireless Networks. Canada: John Wiley \& Sons, Inc., 2014. 
[2] S. N. Sangvikar and A. B. Diggikar, "Femtocell Technology," Journal of Information, Knowledge and Research in Electronics and Communication, Vol. 2, No. 2, pp. 954-962, 2013.

[3] Z. Wei, Z. Feng, Y. Li, and Q. Zhang, "Voronoibased coverage optimization for multifemtocells", In: Proc. of 2010 IEEE International Conference on Wireless Information Technology and Systems, Honolulu, HI, USA, pp. 1-4, 2010.

[4] T.-T. Nguyen, J.-S. Pan, T.-Y. Wu, T.-K. Dao, and T.-D. Nguyen, "Node Coverage Optimization Strategy Based on Ions Motion Optimization", Journal of Network Intelligence, Vol. 4, No. 1, pp. 1-9, 2019.

[5] X. Lu, W. Cheng, Q. He, J. Yang, and X. Xie, "Coverage Optimization Based on Improved Firefly Algorithm for Mobile Wireless Sensor Networks", In: Proc. of 2018 IEEE 4th International Conference on Computer and Communications, Chengdu, China, pp. 899-903, 2018.

[6] T.-T. Nguyen, J.-S. Pan, J. C.-W. Lin, T.-K. Dao, and T.-X.-H. Nguyen, "An Optimal Node Coverage in Wireless Sensor Network Based on Whale Optimization Algorithm", Data Science and Pattern Recognition, Vol. 2, No. 2, pp. 1121, 2018.

[7] Z. Wang, H. Xie, Z. Hu, D. Li, J. Wang, and W. Liang, "Node Coverage Optimization Algorithm for Wireless Sensor Networks based on Improved Grey Wolf Optimizer", Journal of Algorithms \& Computational Technology, Vol. 13, 2019.

[8] M. Arif, S. Wyne, and A. J. Nawaz, "Indoor Localization using Voronoi Tessellation", Advances in Electrical and Computer Engineering, Vol. 18, No. 4, pp. 85-90, 2018.

[9] A. Landström, H. Jonsson, and A. Simonsson, "Voronoi-Based ISD and Site Density Characteristics for Mobile Networks", In: Proc. of 2012 IEEE Vehicular Technology Conference, Quebec City, QC, Canada, pp. 1-5, 2012.

[10] O. A. Akinlabi, B. S. Paul, M. K. Joseph, and H. C. Ferreira, "Indoor Communication: Femtocell Behavior in an Indoor Environment", In: Proc. of the International MultiConference of Engineers and Computer Scientists, Vol. 2, Hong Kong, p. 5, 2015.

[11] T. Zahir, K. Arshad, A. Nakata, and K. Moessner, "Interference Management in Femtocells", IEEE Communications Surveys \& Tutorials, Vol. 15, No. 1, pp. 293-311, 2013.
[12] S. Saleem and H. King, "Avoidance of Co-tier Interference between Femtocells with Different Access Modes", International Journal of Information and Communication Technology Research, Vol. 2, No. 8, p. 10, 2012.

[13] E. Pateromichelakis, M. Shariat, A. U. Quddus, and R. Tafazolli, "On the analysis of co-tier interference in femtocells", In: Proc. of 2011 IEEE 22nd International Symposium on Personal, Indoor and Mobile Radio Communications, Toronto, ON, Canada, pp. 122-126, 2011.

[14] J. N. Portela and M. S. Alencar, "Cellular Coverage Map as a Voronoi Diagram", Journal of Communication and Information Systems, Vol. 23, No. 1, pp. 22-31, 2008.

[15] C. Forbes, M. Evans, N. Hastings, and B. Peacock, Statistical Distributions, Fourth Edition. New Jersey: John Wiley \& Sons, Inc., 2011.

[16] S. Z. Selim and M. A. Ismail, "K-means-type algorithms: A generalized convergence theorem and characterization of local optimality", IEEE Transactions on Pattern Analysis and Machine Intelligence, Vol. 1, pp. 81-87, 1984.

[17] J. P. Ortega, N. N. A. Ortega, A. V. Villalobos, R. P. Rangel, C. Z. Díaz, and A. M. Rebollar, "The K-Means Algorithm Evolution", Introduction to Data Science and Machine Learning, pp. 1-22, 2020.

[18] Q. Du, V. Faber, and M. Gunzburger, "Centroidal Voronoi Tesselations: Applications and Algorithms", Society for Industrial and Applied Mathematics (SIAM) Review, Vol. 41, No. 4, pp. 637 - 676. 1999.

[19] E. K. P. Chong and S. H. Zak, An Introduction to Optimization, $2^{\text {nd }}$ Edition. Canada: John Wiley \& Sons, Inc., 2001.

\section{Appendix A}

\begin{tabular}{|c|l|}
\multicolumn{1}{|c|}{ Tabel 2. List of notations } \\
\hline Notation & \multicolumn{1}{|c|}{ Definition } \\
\hline$U_{x y}$ & $\begin{array}{l}\text { Generated user positions in two- } \\
\text { dimensional space. }\end{array}$ \\
\hline$C_{x y}$ & The FAP locations. \\
\hline$\widehat{V}$ & Voronoi regions. \\
\hline$r_{\min }$ & Initial minimum radius \\
\hline$r_{\max }$ & Initial maximum radius \\
\hline$r_{\text {opt }}$ & Optimum radius \\
\hline$L_{i}$ & Overlaps area of optimum radius \\
\hline$A$ & True coverage of optimum radius \\
\hline$O$ & Blank spots area of optimum radius \\
\hline
\end{tabular}

